\title{
Familial carnitine deficiency: further evidence for autosomal recessive transmission with variable expression
}

\author{
ELI SHAHAR, ${ }^{*} \dagger$ NATAN BRAND, ${ }^{*}$ YEHUDA SHAPIRA, $\ddagger$ VARDA BARASH, $\S$ \\ ALIZA GUTMANß
}

From the Pediatric Neurology Unit, ${ }^{*}$ Department of Pediatrics, Chaim Sheba Medical Center, Tel-Hashomer, Pediatric Neurology Unit $\ddagger$ and Department of Clinical Biochemistry, $\S$ Hadassah Medical Center, Jerusalem, Israel, and the Division of Neurology, $\uparrow$ The Hospital for Sick Children, Toronto, Ontario, Canada

SUMMARY Carnitine deficiency occurring in families has been rarely reported and the genetic transmission has not yet been clearly elucidated. Five members of one family showing marked heterogeneity of carnitine deficiency states are presented. In three patients, there was no correlation between measurable carnitine levels in serum and muscle and the clinical findings. The parents, who@ are remote relatives from an isolated village in Kurdistan (Iraq), had low muscle carnitine levels; $; \stackrel{\mathbb{Q}}{\circ}$ however, they were asymptomatic. One son, with systemic carnitine deficiency causing muscle weak ness and recurrent episodes of severe hepatic encephalopathy, died at 3 years of age. His brother had $\delta$ mild proximal muscle weakness associated with low muscle carnitine levels. He was successfully treated with L-carnitine and prednisone. A daughter is asymptomatic, but with low serum and muscle levels of carnitine. The marked heterogeneity of carnitine deficiency states within one family,; where both parents had low muscle carnitine levels, suggests an autosomal recessive inheritance with variable expression.

Carnitine is a quaternary amine essential for the transport of long-chain free fatty acids (LCFFA) across the inner mitochondial membrane where they undergo $\beta$-oxidation providing the major source of energy to the Krebs cycle and the respiratory chain. ${ }^{1}$ Most of the carnitine required daily is synthesised in the liver and is transported primarily to muscles where LCFFA are the sole source of fuel. Carnitine deficiency states were traditionally classified into either myopathic or systemic forms. The myopathic form is characterised by proximal and truncal muscle

Address for reprint requests: Dr E Shahar, Division of Neurology, The Hospital for Sick Children, Toronto, Ontario, Canada M5G $1 \mathrm{X} 8$.

Received 24 March 1987 and in revised form 20 July 1987. Accepted 27 July 1987 weakness and accumulation of lipid droplets within muscle fibres associated with low muscle carnitine levels. ${ }^{2}$ In systemic carnitine deficiency, carnitine levels are reduced in the liver, serum and muscle resulting in muscle weakness and recurrent episodes of severe hepatic encephalopathy commonly terminating in death. ${ }^{34}$ This concept of differentiation into distinct clinical myopathic and systemic forms was challenged, however, when mixed forms were reported noting the lack of correlation between plasma or muscle carnitine levels and clinical features. ${ }^{5-8}$

Familial carnitine deficiency has been rarely reported, ${ }^{9-11}$ describing marked heterogeneity of carnitine deficiency státes, thus postulating an autosomal recessive mode of transmission. We have studied five members of one family who had various forms of carnitine deficiency. This provides further evidence for an autosomal recessive mode of inheritance with variable expression in familial carnitine deficiency. 


\section{Case reports}

Two brothers and a sister, 6 months, 11 months and 5 years of age at diagnosis, and their parents were investigated. All the children were born at term following an uneventful pregnancy, delivery and post-natal period. Documented mental and cognitive development was normal in all three children. The parents are remote family relatives, both coming from an isolated small community in Kurdistan (Iraq) where many intra-familial marriages occurred.

Total serum carnitine levels were measured in all family members and compared with a control value of $60 \pm 30$ $\mathrm{nmol} / \mathrm{ml}$ established in the laboratory of one of the authors (AG). Muscle biopsies were performed in all five patients studied and specimens were submitted for routine microscopy, histochemistry and electron microscopy. The muscle carnitine levels were measured in a fresh specimen taken from the biopsy and compared with a control value of $2 \cdot 5 \pm$ $1 \mathrm{nmol} / \mathrm{mg}$ wet weight. The total carnitine levels in serum and muscle in relation to the respective clinical presentations are illustrated in the table.

Patient No 1: The index patient was admitted at age 11 months with a second episode of a Reye-like syndrome. Motor milestones were delayed from birth. He completely recovered from a comatose state lasting 36 hours which had occurred 6 weeks prior to this present episode. Three days prior to admission he developed low-grade fever, irritability and recurrent vomiting. He gradually lapsed into a deep coma associated with intractable generalised tonic-clonic seizures and respiratory failure accompanied with hypoglycaemia and impaired liver function tests. High doses of dilantin, phenobarbital, diazepam and paraldehyde were needed to control the intractable seizures. Examination, on admission revealed generalised muscle wasting and hepatomegaly. He was deeply comatose with fixed non-reactive pupils, generalised hypotonia with brisk deep tendon refiexes and extensor plantar responses. Persistent elevation of the intracranial pressure to levels up to $40 \mathrm{~mm} \mathrm{Hg}$ (normal value: up to $15 \mathrm{~mm} \mathrm{Hg}$ ), was managed with a full "brain resuscitation" protocol including fluid restriction, hypothermia, thiopentothal coma, dexamethasone and mannitol therapy. Serum and CSF levels of ammonia and lactic acid were abnormally elevated. The diagnosis of systemic carnitine deficiency was established by total serum and muscle carnitine values, which were as low as $8.8 \mathrm{nmol} / \mathrm{ml}$ and 0.25 $\mathrm{nmol} / \mathrm{mg}$ wet weight respectively. The muscle biopsy demonstrated "ragged-red" fibres, multiple fat droplets within the fibres with a predominance of type I fibres. Abnormal,

Table Total carnitine levels in serum and muscle in relation to clinical state of carnitine deficiency

\begin{tabular}{llll}
\hline Patient & $\begin{array}{l}\text { Serum } \\
(\text { nmol } / \mathrm{ml})\end{array}$ & $\begin{array}{l}\text { Muscle } \\
\text { (nmol/mg } \\
\text { wet weight) }\end{array}$ & $\begin{array}{l}\text { Clinical } \\
\text { presentation }\end{array}$ \\
\hline No. 1 & 8.8 & 0.23 & Systemic \\
No. 2 & 39 & $0 \cdot 26$ & Myopathic \\
No. 3 & 25 & 0.43 & Asymptomatic \\
Mother & 43 & 0.73 & Asymptomatic \\
Father & 74 & 1.03 & Asymptomatic \\
Control & $60 \pm 30$ & 0.89 & \\
\hline
\end{tabular}

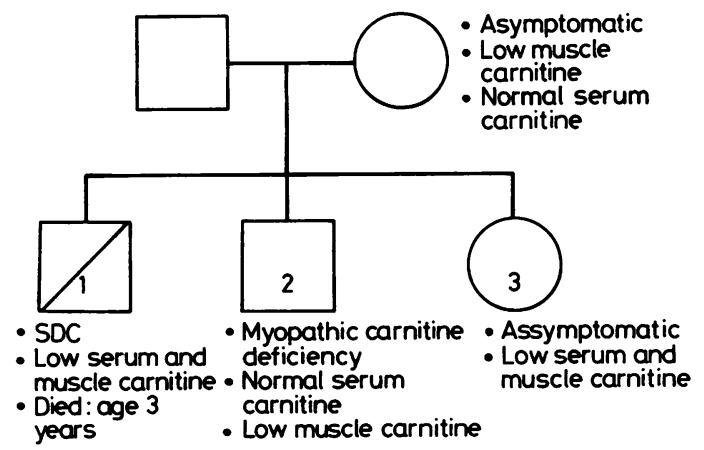

Fig Family pedigree and associated carnitine abnormalities. (SDC = systemic deficiency of carnitine.)

deformed mitochondria were observed on electron microscopy. Treatment was initiated with massive doses of Lcarnitine of $400 \mathrm{mg} / \mathrm{kg}$ which normalised his serum and muscle carnitine levels. However, he remained in a persistent vegetative state with frequent myoclonic seizures and died at age 3 years.

Patient No 2: The younger brother of the proband presented with mild muscle weakness during the first few months of life. At age 5 months he still had a mild head lag and he did not roll over. Examination revealed mild weakness of the neck muscles as well as mild proximal weakness. Serum carnitine levels were normal $(39 \mathrm{nmol} / \mathrm{ml})$. Two consecutive muscle biopsies favoured a lipid storage myopathy. Total muscle carnitine levels were found to be low in both biopsies, being 0.26 and $0.46 \mathrm{nmol} / \mathrm{mg}$ wet weight, respectively. Treatment was begun with a combination of oral L-carnitine and prednisone. His muscle power returned to normal although the muscle carnitine levels remained low. His psychomotor development and muscle power remain normal with a follow-up period of 3 years.

Patient No 3: The asymptomatic sister of patients 1 and 2 was seen at age 5 years. Physical examination revealed normal muscle power. Serum and muscle carnitine levels were found low at $25 \mathrm{nmol} / \mathrm{ml}$ and $0.79 \mathrm{nmol} / \mathrm{mg}$ wet weight, respectively. Muscle biopsy showed type I fibre predominance. She remains asymptomatic with a follow-up period of 3 years.

Patients 4 and 5: Total serum carnitine levels were found to be normal in both parents. Muscle carnitine levels were lower than normal in the mother as well as the father at 1.03 and $0.89 \mathrm{nmol} / \mathrm{mg}$ wet weight, respectively, although they were greater than the carnitine levels of their affected sons. Type I fibre predominance was found only in the mother's muscle biopsy. Both parents are healthy with normal mentation.

\section{Discussion}

Marked heterogeneity of carnitine deficiency states, with respect to clinical presentation or laboratory findings, as documented in the family presented, has not been previously described. This family includes a male infant with systemic carnitine deficiency, his 
brother with muscle carnitine deficiency, a completely asymptomatic sister with abnormally low serum and muscle carnitine levels, and both asymptomatic parents with low muscle carnitine levels. The lack of symptoms despite low serum and muscle levels of carnitine, as noted in the sister, is extremely unusual. A few cases describing patients with low serum and muscle carnitine levels showing only myopathic features have been reported. ${ }^{5-7}$ On the other hand, patients with a clinical presentation of systemic carnitine deficiency and normal serum carnitine levels have also been reported. ${ }^{89}$ The family we have presented helps to demonstrate the difficulties in drawing a clear distinction between carnitine deficiency states including multisystem involvement, isolated muscle carnitine deficiency, or asymptomatic patients despite low serum or muscle carnitine. A muscle biopsy is therefore recommended in all close relatives of carnitine deficient patients, regardless of their clinical status, to search for evidence of lipid storage myopathy.

Familial carnitine deficiency is a very rare condition and the inheritance pattern of this disorder has not been entirely elucidated. To date, only few reports of carnitine deficiency states occurring within families have been published. ${ }^{9-11}$ Scholte et al $^{9}$ presented two sisters, one who died with systemic carnitine deficiency while her sister had only mild muscle weakness despite low serum and muscle carnitine levels. The parents, who were asymptomatic, were found to have normal serum carnitine levels. Muscle biopsies were not performed. Cornelio et $a l^{4}$ reported three cases from an isolated region in the Italian Alps which is reputed to have a high rate of inbreeding. One of the patients was a product of a consanguinous marriage, and therefore one could speculate a familial origin to the systemic carnitine deficiency in this case. They described a second family where both parents had decreased serum levels of carnitine and their two children who developed a fatal "lipid storage myopathy with carnitine deficiency". These authors also report ${ }^{10}$ two identical twins who died at age three months from a metabolic encephalopathy reminiscent of Reye syndrome. One of them was investigated and found to have low carnitine levels in liver, serum and muscle along with a lipid storage myopathy mainly in type I fibres. Both parents, reported to be asymptomatic, were thoroughly investigated including liver and muscle biopsies. Total carnitine levels were low in both with abnormal distribution of carnitine esters in the plasma. Liver carnitine levels were reduced in both, while muscle levels were normal in the mother and probably low in the father. Muscle and liver biopsies in both parents, however, were morphologically normal. Based on their experience, these authors suggest an autosomal recessive mode of inheritance. Recently, Cruse et al, ${ }^{11}$ described two sisters with sys- temic carnitine deficiency demonstrating no correlation between the plasma carnitine levels and the clinical manifestations. Free serum carnitine levels were reduced in both patients although they were two times higher in the asymptomatic one. The parents had normal serum carnitine levels; muscle biopsies were not performed. In both patients a significant abnormality in the renal clearance of carnitine was found; a mild abnormality was present in the mother.

The present description of a family demonstrating marked heterogeneity of familial carnitine deficiency states with a lack of correlation between measurable levels of carnitine in muscle or serum further substantiates previous speculations of an autosomal recessive mode of transmission of this disorder showing variable expression. Similar to the reports of patients living in isolated remote communities in the Italian Alps ${ }^{410}$ the parents in the family we have presented are remote relatives from an isolated Jewish village in the mountains of Kurdistan (Iraq). Thus, the risk of the occurrence of an inborn error of metabolism such as carnitine deficiency is increased in such consanguinous relationships.

\section{References}

1 Bremer J. Carnitine-metabolism and function. Physiol $\operatorname{Rev}$ 1983;63:1420-80.

2 Angel AG, Angelini C. Carnitine deficiency of human skeletal muscle with associated lipid storage myopathy. Science 1973;179:899-902.

3 Karpati G, Carpenter S, Angel AG, et al. The syndrome of systemic carnitine deficiency. Neurology 1975; 25:16-24.

4 Cornelio F, DiDonato S, Feluchetti D, et al. Fatal cases of lipid storage myopathy with carnitine deficiency. $J$ Neurol Neurosurg Psychiatry 1977;40:170-8.

5 Angelini C, Govoni E, Bragaglia MM, Vergani L. Carnitine deficiency: acute postpartum crisis. Ann Neurol 1978;4:558-61.

6 Carrol JE, DeVivo DC, Brook MH, et al. Carnitine "deficiency": Lack of response to carnitine therapy. Neurology 1980;30:618-26.

7 Scarlato G, Pellegrini G, Cerri C, et al. A syndrome of carnitine deficiency: Morphological and metabolic correlations in two cases. Can J Neurol Sci 1978;5:205-13.

8 Ware AJ, Burton WC, McGarry JD, et al. Systemic carnitine deficiency. J Pediatr 1978;93:959-64.

9 Scholte HR, Meijer AEFH, Van Vijngaarden GK, Leenders KL. Familial carnitine deficiency: A fatal case and subclinical state in a sister. J Neurol Sci 1979;42:87-101.

10 DiDonato S, Rimoldi M, Cornelio F, et al. Evidence for autosomal recessive inheritance in systemic carnitine deficiency. Ann Neurol 1982;11:190-2.

11 Cruse RP, DiMauro S, Towfighi J, Trevsian C. Familial systemic carnitine deficiency. Arch Neurol 1984; 41:301-5. 